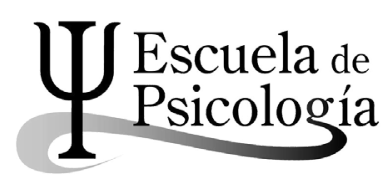

Wímb lu, Rev. electrónica de estudiantes Esc. de psicología, Univ. de Costa Rica. 10 (2): 39-54, 2015 / ISSN: 1659-2107

\title{
EFECTOS DE UN PROGRAMA DE ASIGNATURAS COMPLEMENTARIAS PARA LA FORMACIÓN DE COMPETENCIAS GENÉRICAS SOBRE VARIABLES PSICOLÓGICAS
}

\author{
Effects of a support programme for training of Generic \\ Skills over psychological variables
}

\author{
Gracia Navarro-Saldaña ${ }^{a}$ \\ Marcela Varas-Contreras ${ }^{b}$ \\ Jorge Maluenda-Albornoz ${ }^{c}$ \\ Pablo Arriagada-Pizarro ${ }^{d}$
}

\begin{abstract}
Resumen: La presente investigación analiza el efecto de las Asignaturas Complementarias de la Universidad de Concepción, destinadas a la formación de competencias genéricas, sobre seis variables psicológicas que se les asocian, estas son Asertividad, Empatía, Estilos de afrontamiento, Autoeficacia para Inteligencias Múltiples, Autoatribución de Conductas e Intensiones Socialmente Responsables. Se realiza un estudio descriptivo de un solo grupo, con evaluación pre y post participación en asignaturas complementarias. La muestra se conforma de 40 estudiantes universitarios, 14 hombres y 26 mujeres, quienes cursan desde segundo año de una carrera de pregrado que pertenece al área Químico-Biológica, SocialHumanista o Físico-Matemática. Se aprecian diferencias significativas post participación en asignaturas complementarias en las variables autoeficacia para inteligencia lingüística y espacial, e intenciones socialmente responsables.
\end{abstract}

Palabras clave: Competencias Genéricas; Asertividad; Empatía; Estrategias de Afrontamiento; Autoeficacia para Inteligencias Múltiples; Comportamiento e Intenciones Socialmente Responsables.

a Universidad de Concepción. Psicóloga. Doctora en Educación. Chile. Correo: gnavarro@udec.cl.

b Universidad de Concepción. Ingeniero Civil Informático. Magíster en Ciencias de la Computación. Chile. Correo: mvaras@udec.cl.

c Universidad de Concepción. Psicólogo. Magíster en Política y Gobierno. Chile. Correo: jorgemaluenda@udec.cl.

d Ingeniero Civil Industrial. Universidad de Concepción. Correo: paarriagadap@gmail.com. Recepción: 5/6/2015 Aceptación: 14/7/2015. 


\begin{abstract}
The following study analyzes the effect of Universidad de Concepción's support programme, focused on training of generic skills, over six psychological variables associated to them: Asertivity, Empathy, Coping Styles, Self-efficacy for Multiple Intelligences, Selfattribution of Behavior and Socially Responsable Intentions. It is a single group descriptive study, with pre and post evaluations upon participating in the complementary courses. The sample is comprised of 40 undergraduate students, 14 male and 26 female, who are currently in their second year or higher in an undergraduate program of the QuemicalBiological, Social-Humanist or Physics-Mathematic areas. Significant differences are apparent post participation in complementary courses in the variables of self-efficacy for linguistic and spatial intelligence, in addition to a rise of score in socially responsable intentions.
\end{abstract}

Key Words: Generic Competences; Asertivity; Empathy; Coping Styles; Self-efficacy for Multiple Intelligences; Self-attribution of Behavior and Socially Responsable Intentions.

\title{
Introducción
}

La competencia es entendida como un sistema complejo de conocimiento, creencias y acciones construidos a partir del dominio bien organizado de distintos saberes (Simone, 2002) donde, existe cierto consenso para considerar el conocimiento (saber), la habilidad en las técnicas requeridas (hacer) y la capacidad de desenvolverse (ser) (Calderón, 2012).

Se ha desarrollado la distinción entre Competencias Específicas (CE) y Competencias Genéricas (CG). Las primeras relacionadas con una disciplina de estudio específica y las segundas relacionadas con aquellas comunes a cualquier tipo de programa (González \& Wagenaar, 2006). Mientras que las CE representan las capacidades esenciales requeridas para desempeñar el trabajo con eficacia, las CG se aplican al trabajo en general, más que a tareas específicas de profesiones concretas (Gómez-Mendoza \& Alzate-Piedrahita, 2010).

Se considera a las CG transferibles a distintos contextos (Gilbert, Balatti, Turner, \& Whitehouse, 2004), pudiendo ser utilizadas en el aula de clases pero también en la vida personal y en el ambiente laboral (Burke, 2002). Son transversales a diferentes campos sociales, relevantes en el ámbito académico, profesional, personal y social (Rychen \& Salganik, 2003), involucrando los conocimientos, habilidades y actitudes que un individuo posee (Kallioinen, 2010), pudiendo ser utilizadas en diferentes situaciones, principalmente, porque facilitan que las personas puedan seguir aprendiendo durante toda la vida, a diferencia de las CE que se piensa podrían ser obsoletas en algún momento debido a los cambios tecnológicos y sociales (Corominas, 2001). Son además, multifuncionales, puesto que son requeridas en un amplio y diverso campo de requerimientos cotidianos, profesionales y de la vida social, además de ser multidimensionales debido a que consideran dimensiones perceptivas, normativas, cooperativas y conceptuales (Rychen \& Salganik, 2003). 
La Universidad de Concepción (UdeC) de Chile, cuenta con un modelo educativo propio, para formar en cuatro grandes CG. Por su amplitud, complejidad y las subcompetencias incluidas en cada una, se habla de macro-competencias genéricas y las define como sigue a continuación:

Pensamiento Crítico: "Pensamiento de orden superior, autónomo y activo, que se orienta al análisis sistemático de las necesidades del medio social y ambiental. Integra la perspectiva personal, la de otros y la información proveniente del medio en el análisis de la información, la interpretación, la reflexión acerca de la información, le evaluación de las implicancias y consecuencias de los actos, las explicaciones de los fenómenos la emisión de juicios fundamentados en pos de un propósito definido (UdeC, 2015, p.7).

Comunicación: "Capacidad de expresar con claridad, coherencia y precisión las ideas, conocimientos y sentimientos, adecuándose a diferentes contextos de acuerdo a las características de la audiencia a la cual se dirige. Involucra la validación de los interlocutores y la utilización de estrategias de comunicación verbal, oral, escrita y no verbal, que permiten integrar las intenciones del por qué decimos lo que decimos y como lo decimos con el fin de mantener un diálogo crítico y constructivo" (UdeC, 2015, p.12). El modelo considera la comunicación principalmente desde la perspectiva del hablante, sin embargo su desarrollo considera los aspectos relacionados con el interlocutor como su carácter relacional, la relevancia de la asertividad, el papel de la escucha activa, entre otros. Estos no son el centro de los objetivos educativos de este modelo los que han sido elaborados manteniendo prudencia de su posibilidad de realización teniendo en consideración las características de los estudiantes y de la institución.

Trabajo en equipo interdisciplinario y emprendimiento: "Implica la capacidad personal para descubrir oportunidades, desafíos y entregar respuestas innovadoras ante las propias necesidades y necesidades de otros, valorando la importancia del trabajo colaborativo e interdisciplinario como herramienta necesaria para alcanzar los objetivos" (UdeC, 2015, p.16).

Responsabilidad Social: "Intención, capacidad y obligación de responder ante la sociedad, por acciones u omisiones profesionales que impactan al bien común. Requiere de la conciliación de la satisfacción de las propias necesidades con el aporte a la satisfacción de las necesidades de los demás. Se manifiesta en un actuar responsable en proyectos de distinta índole, relacionadas con la propia competencias profesional y académica, en orientación de las actividades individuales y colectivas en un sentido que contribuya, desde el servicio, a generar equidad para el desarrollo de todos; en comportamientos de cooperación, participación, autocontrol y compromiso ciudadano" (UdeC, 2015, p.20).

Las MCG del modelo educativo de la Universidad de Concepción Chile, a partir de sus distintos subcomponentes, involucran aspectos relacionados con distintas variables psicológicas, las que se especifican a continuación. 
La empatía es una respuesta afectiva de comprensión sobre el estado emocional de otros, que induce a sentir el estado en que se encuentra el otro (Eisenberg et al., 2006). Al incluir tanto respuestas emocionales (dimensión emocional) como la capacidad para entender los estados afectivos de los demás (dimensión cognitiva) (Gutiérrez, Escartí, \& Pascual, 2011), es posible observar un campo común entre las MCG y la empatía, en tanto se necesita el desarrollo de estas dos dimensiones para el ejercicio pleno de las MCG.

La asertividad se entiende como el conocimiento y la expresión de los deseos, valores, necesidades, expectativas y disgustos de un sujeto. Requiere evitar los comportamientos agresivos y pasivos (Casas-Anguera, et al., 2014), así como del conocimiento y aceptación de capacidades y limitaciones personales (Velásquez, Arellanez, \& Martínez, 2012).

Por su parte, los estilos de afrontamiento, se entienden como "aquellos esfuerzos cognitivos y conductuales constantemente cambiantes que se desarrollan para manejar las demandas específicas externas o internas que son evaluadas como excedentes o desbordantes de los recursos del individuo" (Lazarus \& Folkman, 1991, p.164), lo que permite gestionar un adecuado afrontamiento frente a la fuente de estrés y afectación emocional (Pérez-Sánchez, Poveda-Serra, \& Gilar-Corbí, 2010). Ambas variables, comparten campo común con las MCG, especialmente, en las dimensiones de la comunicación itra e interpersonal, la capacidad para evaluar y realizar juicios, así como el autocontrol, todos, aspectos componentes de la cuatro MCG.

La confianza para realizar exitosamente actividades relacionadas con las ocho inteligencias múltiples propuestas por Gardner o Autoeficacia para Inteligencias Múltiples (Pérez, Beltramino, \& Cupani, 2003) presenta especial interés en relación a las MCG UdeC, especialmente en sus dimensiones intra e interpersonal donde se aprecia mayor vinculación teórica. Esto debido a la observada relación entre la confianza de los alumnos en cuanto a su capacidad en torno a las Inteligencias Múltiples y el desempeño de los alumnos en el proceso de enseñanza-aprendizaje (Pérez \& Medrano, 2007).

La Conducta Socialmente Responsable se refiere a aquellas conductas morales (Prosociales, cooperativas y de autocontrol) que tienen a la base una intención orientada hacia el bien común; intención que implica alcanzar la capacidad de conciliar la satisfacción de las propias necesidades con la satisfacción de las necesidades de otros (Navarro, 2012). Esta privilegia las acciones sobre el bien común, las necesidades de un grupo y las relaciones con el entorno (Arango et al., 2014). La Autoatribución de Conductas e Intensiones Socialmente Responsables es una dimensión compuesta por la autopercepción acerca de la realización de conductas socialmente responsables y sobre la intensión de efectuarlas, subdimensión de la MCG Responsabilidad Social que además contribuye al desarrollo de las demás MCG. 
El modelo educativo de la Universidad de Concepción de Chile, contempla la formación en CG a través de su incorporación en asignaturas disciplinares e integradoras de las carreras, y en asignaturas complementarias que son en las que se centra esta investigación.

Son asignaturas de inscripción voluntaria, no forman parte de la programa de estudios, y forman a los alumnos en contenidos de disciplinas diferentes a la que estudian, de manera integrada al desarrollo de las Macrocompetencias Genéricas (MCG) del modelo UdeC.

Su oferta se encuentra conformada al año 2014 por 10 asignaturas con régimen semestral y 14 horas cronológicas de trabajo en aula, planificadas e impartidas por especialistas en un área disciplinar, quienes implementan el programa de la asignatura utilizando metodologías basadas principalmente en 3 enfoques de enseñanza vinculados al desarrollo de competencias genéricas.

Las asignaturas complementarias UdeC se basan en el Enfoque de Aprendizaje Centrado en el Estudiante. Este promueve el rol activo del estudiante en su propio aprendizaje ofreciéndole la posibilidad de aprender a aprender, o dicho de otra manera, adquirir las herramientas necesarias para organizar y autorregular los propios aprendizajes (Corominas, 2001; Cerezo, et al., 2010; Hernández, Sales, \& Cuesta, 2010). Se reconoce que un aprendizaje de calidad exige que los alumnos adquieran la capacidad para reflexionar sobre su propia actividad de aprendizaje y adaptarla en función de los diferentes contextos (Hernández et al., 2010). Además, requiere un docente activo que utilice metodologías que creen situaciones y problemas donde los estudiantes puedan desarrollar las competencias esperadas (Torra-Bitlloch, De Corral, \& Pérez, 2012) con un rol de acompañamiento y guía a través del proceso, validando al alumno como un aprendiz activo y autorregulado (Tigelaar, 2004).

Las asignaturas complementarias UdeC se basan además en el Enfoque de Evaluación Auténtica donde el proceso evaluativo forma parte de la enseñanza involucrando procesos cognitivos y socio-afectivos (Díaz \& Barroso, 2014) contextualizando los aprendizajes para una experiencia con sentido, tanto en el plano personal, interpersonal como el laboral (Vallejo \& Molina, 2014).

La evaluación permitirá planificar y programar la enseñanza a realizar (Función orientadora), detectar el progreso de los estudiantes y tomar medidas que permitan disminuir sus debilidades así como potenciar sus fortalezas (Función formativa), comprobando además, los resultados alcanzados y valorando el grado de consecución de los mismos (Función Sumativa) (Correa, Puerta, \& Restrepo, 2002). Desde aquí, tanto la autoevaluación como la evaluación entre iguales adquieren mucha importancia como herramientas para la reflexión permanente, la retroalimentación en el proceso de enseñanza y la autorregulación del aprendizaje (Díaz \& Barroso, 2014). La evaluación de pares ha mostrado efectividad en promover la colaboración incrementando su intencionalidad 
formativa, fomentando el diálogo, la interacción enriquecedora y la creación de significados comunes con compañeros y docentes (Ibarra, Rodríguez, \& Gómez, 2012). Los alumnos la evalúan positivamente ambos métodos por su valor en ayudar a mejorar los procesos y productos del aprendizaje, y el desarrollo de estrategias interpersonales, permitiendo desarrollar la capacidad para realizar juicios y evaluar a otros (Ibarra, Rodríguez, \& Gómez, 2012).

Las asignaturas complementarias UdeC se basan en el Enfoque del Aprendizaje Colaborativo, el que permite organizar el proceso de enseñanzaaprendizaje a partir de una mayor focalización en las responsabilidades colectivas y en liderazgos compartidos (González \& García, 2007) además de la puesta en común de las experiencias de los distintos alumnos, así como el mutuo y constante enriquecimiento.

En un contexto colaborativo existe una aceptación por parte de los miembros del grupo de la responsabilidad de las acciones y decisiones del grupo, siendo cada integrante responsable de su propio aprendizaje y a la vez, del aprendizaje de los restantes miembros del grupo (Iborra \& Izquierdo, 2010). La estrategia no alcanza su objetivo de no existir a la base intencionalidad y planificación que permitan el logro de los aprendizajes deseados (Lillo, 2013), haciendo al trabajo grupal necesario pero no suficiente. Ha mostrado eficacia en la formación de actitudes y valores, la mejora de la motivación escolar, la práctica de la conducta prosocial, la pérdida progresiva de egocentrismo, el desarrollo de una mayor independencia y autonomía (González \& García, 2007), además de favorecer la participación y motivación de los alumnos, el desarrollo de competencias y la mejora de los aprendizaje alcanzados (Lillo, 2013).

La presente investigación busca observar el efecto de las asignaturas complementarias sobre las variables psicológicas previamente presentadas. Además, se pretende caracterizar al grupo de estudiantes que cursa estas asignaturas complementarias observando la emergencia de diferencias por sexo y área disciplinar (físico-matemático, químico-biológico y socio-humanista), además de apreciar las relaciones entre las distintas variables psicológicas.

En relación a lo anterior, se plantean las siguientes hipótesis de trabajo: 1) No existen diferencias de sexo en cuanto autoeficacia para inteligencias múltiples y estilo de afrontamiento, entre los estudiantes que inscriben asignaturas complementarias asociadas a las competencias genéricas UdeC; 2) Existen diferencias de sexo en autoatribución de comportamiento e intenciones socialmente responsables, en empatía y asertividad para este grupo de estudiantes; 3) Existen diferencias según área disciplinar en autoeficacia para inteligencias múltiples, estilo de afrontamiento y en autoatribución de comportamiento e intenciones socialmente responsables, en empatía y asertividad para estos estudiantes; 4) Existen diferencias en autoatribución de comportamiento en intenciones socialmente responsables antes y después 
de participar en una asignatura complementaria, pero no existen diferencias significativas en las otras variables estudiadas.

\section{Método}

\section{Participantes}

La muestra se conforma de un grupo de 40 estudiantes universitarios, quienes de forma voluntaria se inscriben para cursar alguna de las asignaturas complementarias ofrecidas por el Programa de Competencias Genéricas de la Universidad de Concepción (UdeC). Estos se distribuyen en 14 hombres (35\%) y 26 mujeres (65\%) quienes cursan desde segundo año de su carrera de pregrado. 16 de estos estudiantes pertenecen a carreras del área Químico-Biológica (40\%); 13 a carreras del área Social-Humanista (32.5\%) y 11 a carreras del área Físicomatemática (27.5\%).

\section{Instrumentos}

Inventario de Autoeficacia para Inteligencias Múltiples (IAMI-R). Desarrollado a partir de su versión original (el IAMI), mide la confianza para realizar exitosamente actividades relacionadas con las ocho inteligencias múltiples propuestas por Gardner (1999) y está compuesto por ocho escalas obtenidas mediante análisis factorial exploratorio en 48 ítems, seis por cada escala. Esto son Inteligencia lingüística, lógico-matemática, Espacial. Musical, Interpsonal, Cinestésica, Intrapersonal y Naturalista (Pérez, Beltramino, \& Cupani, 2003). Ha mostrado una consistencia interna elevada con un Alfa de Cronbach sobre .86, además de apreciarse validez y utilidad predictiva (Lent, Brown, \& Hackett, 1994).

Asertividad. Gambrill \& Richey. Conformado por dos Subescalas - Grado de Incomodidad (GI) y Probabilidad de Respuesta (PR) - que incluyen un total de 39 reactivos (Castaños, Reyes, Rivera \& Díaz, 2011). Presenta situaciones de interacción con extraños, amigos, compañeros de trabajo y personas íntimamente relacionadas. En la adaptación del instrumento se observa una alta confiabilidad medida a través del coeficiente Alfa de Cronbach, .89 para la subescala Grado de Incomodidad y .94 para Probabilidad de Respuesta, además de una alta validez de constructo del inventario (Castaños et al., 2011).

El Inventario Estrategias de afrontamiento (CSI). Desarrollado por Tobin, Holroyd, Reynolds \& Kigal (1989) se divide en 2 partes, en la primera, la persona describe lo más detalladamente posible una situación estresante, y la segunda, compuesta por 40 ítems que son respondidos por la persona en una escala Likert de cinco puntos, de acuerdo a la frecuencia con que realiza la situación descrita. 
Al final de la escala se contesta a un ítem adicional acerca de la autoeficacia percibida del afrontamiento, el cual también se responde de acuerdo a una escala Likert (Nava et al., 2010). Ha mostrado adecuadas propiedades psicométricas, con coeficientes de consistencia interna de .63 y .89, y una validez convergente suficiente con todas las medidas utilizadas (Cano, Rodríguez, \& García, 2007).

Índice de reactividad Intepersonal de Davis (Interpersonal Reactivity Index). Esta escala, adaptada al español, consta de 28 ítems con una modalidad de respuesta tipo Likert de 5 alternativas, destinadas a medir la empatía en la persona. Estos ítems se encuentran clasificados a partir de cuatro subescalas a) Fantasía o la tendencia de los sujetos a identificarse con personajes ficticios como los de libros y películas; b) Toma de perspectiva o la tendencia/habilidad de los sujetos para adoptar la perspectiva o punto de vista de otras personas; c) Preocupación empática o la tendencia de los sujetos a experimentar sentimientos de compasión y preocupación hacia otros; y d) Estrés personal o sentimientos de incomodidad y ansiedad al ser testigos de experiencias negativas de otros (Pérez-Albéniz et al., 2003). En cuanto a los niveles de Consistencia Interna de la escala (PérezAlbéniz at al., 2003) observaron un Alfa de Cronbach entre .70 y .78, con una adecuada estructura factorial en sus análisis.

Autoatribución de comportamientos e intenciones socialmente responsables (CSRp). Desarrollado por Davidovich, Espina, Navarro \& Salazar (2005) se compone de 2 escalas que miden 3 dimensiones. Estas son Participación Religiosa, Participación Social y Respeto por el entorno. La primera, mide la auto atribución de frecuencia de conductas socialmente responsables, a partir de la presentación de 30 conductas ante las cuales el sujeto debe responder en una escala Likert con cinco alternativas mientras que, la segunda, evalúa la intención subyacente a cada uno de los 30 comportamientos anteriores, presentándolos nuevamente y enseñando con esto tres alternativas de intención (orientación hacia el beneficio propio, de otros y de todos), a lo que se agrega una cuarta alternativa para aquellos que no pueden identificar una intención definida para ejecutar la conducta (Davidovich et al., 2005). Este instrumento, mostró en el estudio original un Coeficiente Alpha de Cronbach de .82 para la Escala 1 y de .76 para la Escala 2, lo que muestra la adecuada consistencia interna de las escalas Davidovich et al., 2005).

\section{Diseño y procedimiento}

Se realiza un estudio descriptivo de un solo grupo, con evaluación pre y post participación en las asignaturas complementarias. Se carece de grupo de comparación puesto que no es posible, en el contexto de este programa, mantener alumnos fuera de la intervención sin afectar arbitrariamente sus posibilidades equitativas de acceso a la educación. Por ello, se ha debido prescindir de ellas en respecto de los aspectos éticos relacionados a la investigación con personas. 
Los instrumentos fueron aplicados al inicio de las asignaturas complementarias, impartidas el primer semestre de 2014, durante los 7 primeros días y luego, durante los últimos 7 días de la misma, ambas instancias en formato electrónico.

Se realiza análisis univariado para describir las características de la muestra respecto de cada una de las variables estudiadas, además de un análisis bivariado, con un estudio correlacional, para establecer la relación entre variables y observar diferencias en ellas luego de la participación en alguna de las asignaturas descritas.

\section{Resultados}

De los 40 alumnos que inscribieron asignaturas complementarias del programa de Competencias Genéricas UdeC, se pueden determinar las siguientes características en relación a las variables psicológicas estudiadas.

En promedio, el nivel de autoeficacia para realizar exitosamente actividades relacionadas con la inteligencia interpersonal es lo que más predomina en los alumnos, para ambos sexos y pertenecientes a las áreas químico-biológica y social-humanista. Los alumnos del área físico-matemática sin embargo, presentan mayor confianza para realizar actividades relacionadas con la inteligencia lógico matemática.

Respecto de la variable empatía, ambos sexos y las tres áreas disciplinarias coinciden en la preocupación empática relacionada con tendencia a experimentar sentimientos de compasión y preocupación por los demás, como la de mayor manifestación.

En cuanto a las estrategias de afrontamiento, en promedio, la que más se refleja en los alumnos es la resolución de problemas, concordando áreas disciplinarias y sexo de los alumnos.

Los resultados en asertividad muestran que, de acuerdo al grado de incomodidad de los alumnos frente a ciertas situaciones, en promedio, lo que más incomoda a las mujeres es manejar situaciones molestas, mientras que a los hombres les es más incómodo iniciar interacciones. En este mismo aspecto, en promedio, los alumnos de las áreas químico-biológica y social-humanista coinciden con las mujeres, siendo más incómodo para ellos manejar situaciones molestas, mientras que los alumnos del área físico-matemática, concordando con los hombres manifiestan, mayor incomodidad para iniciar interacciones.

En cuanto al grado de probabilidad de respuesta, en promedio, lo que rara vez harían los alumnos corresponde a situaciones que no se relacionan con los factores del inventario de asertividad de Gambrill y Richey II, como resistirse a comprar algo en oferta o prestarle el automóvil a alguien que se los solicita, donde no se aprecian diferencias de sexo. Concuerdan en este mismo aspecto los alumnos de las áreas químico-biológica y social-humanista mientras que, los 
alumnos del área físico-matemática manifiestan que iniciar interacciones es lo que rara vez harían.

Finalmente, respecto de la autoatribución del comportamiento socialmente responsable, en promedio, de igual forma para ambos sexos y las tres áreas disciplinares, los alumnos manifiestan realizar con mayor frecuencia los comportamientos relacionados con el respeto por el entorno.

Respecto de la intención del comportamiento, en la presente investigación los alumnos obtuvieron 76.9 puntos en promedio de un total posible de 120 puntos. Las mujeres alcanzan una puntuación promedio de 75.27 y los hombres 74.23. En cuanto al área disciplinaria, en promedio, los alumnos con mayor intención socialmente responsable son, los alumnos del área social- humanista, con 78.23 puntos, seguidos de los alumnos del área físico-matemática con 74.9 y finalmente los alumnos del área químico-biológica con 72.25 puntos.

Se realizó una comparación de medias de ambas muestras mediante una prueba estadística $t$, previa realización de la prueba de KolmogórovSmirnov para cada variable, para el tratamiento de los datos asimilado a una distribución normal.

Para las variables que no siguieron esta distribución, se realizó la prueba Wilcoxon de rangos con signo para identificar si existe diferencia significativa en las medias. En este caso, ninguna de las variables sometidas a esta prueba resultó ser significativa.

De esta forma, las variables con una significancia asintótica (valor p) $<.05$ o que se encuentran en un intervalo de confianza al $95 \%$ que no incluye al 0 , significa que las variables son estadísticamente significativas y las medias son distintas.

Para las variables psicológicas estudiadas, se presenta evidencia estadística que dentro de los tipos de inteligencia, la inteligencia lingüística e inteligencia espacial presenta diferencias estadísticamente significativas pre y post curso. Esto quiere decir, que existe diferencia en las medias de los datos antes y después de cursar las asignaturas complementarias, a diferencia del tipo de inteligencia lógico matemática, cenestésica, intrapersonal y naturalista, donde no se aprecia diferencia significativa en las medias.

\section{TABLA 1.}

Variables Significativas respecto del Tipo de Inteligencia

\begin{tabular}{|c|c|c|c|c|}
\hline \multirow[t]{2}{*}{ Tipo de inteligencia } & \multicolumn{2}{|c|}{$\begin{array}{l}\text { Intervalo de Confianza al } 95 \% \text { de la diferencia } \\
\text { Pre y Post test }\end{array}$} & \multirow[t]{2}{*}{$\mathrm{T}$} & \multirow[t]{2}{*}{ TSig. } \\
\hline & Inferior & Superior & & \\
\hline Inteligencia Lingüística & -.41 & -.049 & -2.61 & .015 \\
\hline Inteligencia Espacial & -.12 & -2.54 & -2.54 & .018 \\
\hline
\end{tabular}


De esta forma, como se muestra en la Tabla 1, existe impacto significativo en la inteligencia lingüística y espacial de quienes inscribieron asignaturas complementarias del Programa de Competencias Genéricas UdeC.

En relación a empatía y estrategias de afrontamiento, los datos presentados no muestran significancia estadística. De esta forma, se puede decir que el impacto sobre estas variables no es significativo.

Por otro lado, en el caso de la asertividad, la acción de rechazar peticiones de alguien para tener alguna cita o reunión es significativa, por lo que existe impacto sobre la asertividad en los alumnos que cursaron asignaturas complementarias asociadas a las competencias genéricas UdeC.

TABLA 2.

Variables significativas correspondientes a Asertividad

\begin{tabular}{lcccc}
\hline Factor & \multicolumn{2}{l}{$\begin{array}{l}\text { Intervalo de Confianza al } 95 \% \text { de la } \\
\text { diferencia Pre y Post test }\end{array}$} & T & Sig. \\
\hline & Inferior & Superior & & \\
\hline Rechazar Peticiones & .023 & .39 & 2.33 & .029 \\
\hline$* \mathrm{p}<.05$ & & & &
\end{tabular}

Las correlaciones de Pearson son altas y significativas en la mayoría de los datos, mas no se presentó correlación entre la estrategia primaria (Evitar problemas) respecto de las estrategias del comportamiento. Tampoco en las acciones que no corresponden a los factores de las subescala de Probabilidad de Respuesta en el inventario de asertividad.

TABLA 3.

Significancia Intención Socialmente Responsable

\begin{tabular}{lcccc}
\hline Variable & \multicolumn{2}{c}{$\begin{array}{l}\text { Intervalo de Confianza al 95\% de la diferencia } \\
\text { Pre y Post test }\end{array}$} & T & Sig. \\
\hline \multicolumn{2}{c}{ Inferior } & Superior & & \\
\hline $\begin{array}{l}\text { Intención } \\
\text { Responsable }\end{array}$ & Socialmente-5.23 & -.85 & $-2,87$ & .009 \\
\hline
\end{tabular}

$* \mathrm{P}<.05$

Finalmente, en relación a la autoatribución de comportamiento e intenciones socialmente responsables, los datos que corresponden a la autoatribución de frecuencia de conductas socialmente responsables divididas en las dimensiones religiosa, social y el respeto por el entorno, no presentaron significancia estadística. Sin embargo, en relación a la intención socialmente responsable, en promedio, los alumnos alcanzaron un puntaje de 80 , mayor al puntaje inicial al momento de inscribir las asignaturas complementarias. 


\section{Conclusiones y discusión}

A partir de los resultados obtenidos es posible apreciar diferencias de sexo en cuanto asertividad. Por el contrario, no se observaron diferencias de sexo en cuanto a empatía, estilos de afrontamiento y autoatribución del comportamiento e intenciones socialmente responsables. Esto puede relevante para la educación de profesionales al considerar aquellas dimensiones que requieran oferta de formación diferenciada y/o especializada.

Se observan perfiles disímiles en relación al área disciplinar, donde los alumnos difieren en cuanto a autoeficacia para inteligencias múltiples, asertividad y autoatribución de comportamiento e intenciones socialmente responsables. Sin embargo, existe convergencia en cuanto a empatía y estilos de afrontamiento.

En relación a la asertividad los alumnos de las áreas químico-biológica y social-humanista convergen en una mayor incomodidad para manejar situaciones molestas mientras que, los alumnos del área físico-matemática muestran mayor dificultad para iniciar interacciones.

En el caso de la autoeficacia para inteligencias múltiples se observó una alta autoeficacia de las áreas químico-biológica y social-humanista en inteligencia interpersonal mientras que, los alumnos del área físico-matemática en inteligencia lógico-matemática.

En la última variable, la diferencia se encuentra en los puntajes obtenidos por los alumnos de distintas áreas disciplinares donde los alumnos del área social-humanista obtienen un puntaje más alto en intensiones socialmente responsables diferenciándose del más bajo en casi 6 puntos. En estas dos últimas variables, la diferencia puede encontrar explicación en base a características propias del área de desempeño como producto de la formación profesional ofrecida y/o la vocación personal. De cualquier modo, la información anterior será importante en la educación de profesionales teniendo en cuenta aquellas dimensiones en las que puede ser necesario ofrecer formación diferenciada de modo de entregar herramientas que permitan un despliegue apropiado de los alumnos en todas las dimensiones.

Respecto de la participación en las asignaturas complementarias no se observó un efecto sobre empatía y estilos de afrontamiento. Sin embargo, se aprecia un efecto significativo sobre las variables autoeficacia para inteligencias múltiples y asertividad. En el caso de la primera, ocurre un aumento de la autoeficacia en inteligencia lingüística y espacial mientras que, en la segunda se aprecia un aumento en la capacidad para rechazar peticiones para asistir a una cita o reunión.

Los efectos reportados agregan evidencia sobre la posibilidad de formar profesionales, con actividades basadas en la formación centrada en el estudiante, el aprendizaje colaborativo y la evaluación auténtica, que provean efectos sobre 
dimensiones psicológicas. En este caso, la fórmula utilizada para la formación tuvo impacto sobre dimensiones específicas sin perjuicio que otras fórmulas puedan tener efectos significativos sobre estas y otras variables.

Con ello, el diseño presentado tiene efectos positivos en los estudiantes sobre lo que piensan respecto de sus capacidades intelectuales (valoración personal), así como sobre su manera de abordar situaciones (habilidad interpersonal).

No se aprecian diferencias significativas pre-post asignaturas complementarias en torno a autoatribución de conductas socialmente responsables. Sin embargo, se aprecian diferencias significativas en torno a intensiones socialmente responsables, donde existe un aumento de los puntajes en el post test. La disminución de la autoatribución de conductas socialmente responsables puede deberse a una mayor precisión conceptual adquirida a través de las asignaturas complementarias que facilite discriminar de mejor modo en el post test, disminuyendo así los puntajes. Ambos efectos, aparecen en la dirección esperada lo que agrega sustento a la posibilidad de formar profesionales con impacto en dimensiones psicológicas aumentando las expectativas en el campo de la formación en competencias genéricas para la dimensión moral.

\section{Referencias}

Arango, O., Clavijo, S., Puerta, I., \& Sánchez, J. (2014). Formación académica, valores, empatía y comportamientos socialmente responsables en estudiantes universitarios. Revista de la Educación Superior, 43(169), 89-105.

Burke, P. (2002). The development of key skills in higher education. Learning and Teaching Support, 1-17.

Calderón, M. (2012). Competencias genéricas en enfermeras/os titulados de la universidad Arturo Pratt, sede victoria, 2010. Ciencia y Enfermería, 18(1), 89-97.

Cano, F., Rodríguez, L., \& García, J. (2007). Adaptación española del Inventario de Estrategias de Afrontamiento. Actas Españolas de Psiquiatría, 35(1), 11.

Casas-Anguera, E., Prat, G., Vilamala, S., Escandell, M., García-Franco, M., Martin, J., . . . Ochoa, S. (2014). Validación de la versión española del inventario de asertividad Gambrill y Richey en población con dianóstico de esquizofrenia. Anales de Psicología, 30(2), 431-437.

Castaños, S., Reyes, I., Rivera, S., \& Díaz, R. (2011). Estandarización del inventario de asertividad de Gambrill and Richey - II. Revista Iberoamericana de Diagnóstico y Evaluación Psicológica, 29(1), 27-50. 
Cerezo, R., Núñez, J., Rosario, P., Valle, A., Rodríguez, S., \& Bernardo, A. (2010). New media for the promotion of self-regulated learning in higher education. Psicothema, 22(2), 306-315.

Corominas, E. (2001). Competencias genéricas en la formación universitaria. Revista de Educación, 325, 299-321.

Correa, S., Puerta, A., \& Restrepo, B. (2002). Investigación evaluativa. Colombia: ARFO.

Davidovich, M., Espina, A., Navarro, G., \& Salazar, L. (2005). Construcción y estudio piloto de un cuestionario para evaluar comportamientos socialmente responsables en. Revista de Psicología, 14(1), 125-139.

Díaz, F., \& Barroso, R. (2014). Diseño y Validación de una propuesta de evaluación auténtica de competencias en un programa de formación de docentes de educación básica en México. Perspectiva Educacional, 53(1), 36-56.

Eisenberg, N., Zhou, Q., Spinrad, T., Valiente, C., Fabes, R., \& Liew, J. (2006). Relations among positive parenting, children's effourtul control, and externalizing problems: A three-wave longitudinal study. Child Development, 76(5), 1055-1071.

Gardner, H. (1999). Intelligence reframed. Multiple intelliigences for the 21st century. New York: Basic Books.

Gilbert, R., Balatti, J., Turner, P., \& Whitehouse, H. (2004). The generic skills in research higer degrees. Higer Education Research, 23(3), 375-388.

Gómez-Mendoza, M., \& Alzate-Piedrahita, M. (2010). La alegre entrada y el irresistible ascenso de las competencias en la universidad Educación y Educadores. Educación y Educadores, 13(3), 453-474.

González, N., \& García, M. (2007). El Aprendizaje Cooperativo como estrategia de Enseñanza-Aprendizaje en Psicopedagogía (UC): repercusiones y valoraciones de los estudiantes. Revista Iberoamericana de Educación, (42), 6-10.

González, J., \& Wagenaar, R. (2006). Tuning educational structures in Europe. Bilbao: Tuning Project.

Gutiérrez, M., Escartí, A., \& Pascual, C. (2011). Relaciones entre empatía, conducta prosocial, agresividad, autoeficacia y responsabilidad personal y social de los escolares. Psicothema, 23(1), 13-19. 
Hernández, F., Sales, P., \& Cuesta, J. (2010). Impacto de un programa de autorregulación del aprendizaje en estudiantes de grado. Revista de Educación, 353, 571-588.

Ibarra, M., Rodríguez, G., \& Gómez, M. (2012). La evaluación entre pares: Beneficios y estrategias para su práctica en la universidad. Revista de Educación, (359), 206-231.

Iborra, A., \& Izquierdo, M. (2010). ¿Cómo afrontar la evaluación del aprendizaje colaborativo? Una propuesta valorando el proceso, el contenido y el producto de la actividad grupal. Revista General de Información y Documentación, 20, 221-241.

Kallioinen, O. (2010). Defining and comparing generic competences in higher education. European Educational Research Journal, 9(1), 56-58.

Lazarus, R., \& Folkman, S. (1991). Estrés y procesos cognitivos. Barcelona: Martínez Roca.

Lent, R., Brown, S., \& Hackett, G. (1994). Toward a unifing social cognitive thheory of career and academic interest: Choice and performance. Journal of Vocational Behavior, (45), 79-1222.

Lillo, F. (2013). Aprendizaje Colaborativo en la Formación Universitaria de Pregrado. Revista de Psicología, 2(4), 109-142.

Nava, C., Ollua, P., Zaira, C., \& Soria, R. (2010). Inventario de Estrategiass de Afrontamiento: una replicación. Psicología y Salud, 20(2), 213-220.

Navarro, G. (2012). Moralidad y Responsabilidad Social: Bases para su desarrollo y educación. Concepción: Universidad de Concepción.

Pérez, E., \& Medrano, L. (2007). Inventario de autoeficacia para las inteligencias múltiples revisado: Un estudio de validez de criterio. Avances en Medición, 5, 105-114.

Pérez, E., Beltramino, C., \& Cupani, M. (2003). Inventario de Autoeficacia para Inteligencias Múltiples: Fundamentos Teóricos y Estudios Psicométricos. Evaluar, 35-60.

Pérez-Albéniz, A., De Paul, J., Etxeberría, J., \& Montes, M. y. (2003). Adapatación de Interppersonal Reactivity Index (IRI) al Español. Psicothema, 15(2), 267-272. 
Pérez-Sánchez, A., Poveda-Serra, P., \& Gilar-Corbí, R. (2010). Efectos del aprendizaje colaborativo en el uso de estrategias de afrontamiento. Revista Lationamericana de Psicología, 42(3), 481-492.

Rychen, S., \& Salganik,L. (2003). A holistic model of competence. In D. Rychen \& L. Salganik (Eds.), Key Competences for a successful life and well-functioning society (pp.41-62). Gottingen: Hogrefe \& Huber.

Simone, D. (2002). Definition and selection of Key Competencies. Geneva: Hogrefe y Huber Publisher.

Tigelaar, D. (2004). The development and validation of a framework for teaching competences in higher education. Higher Education, 48(2), 253-268.

Torra-Bitlloch, I., De Corral, I., \& Pérez, M. (2012). Identificación, desarrollo y evaluación de competencias docentes en la aplicación de planes de formación dirigidos a profesorado universitario. Revista de Docencia Universitaria, $10(2), 21$.

Universidad de Concepción, UdeC. (2015). Modelo de enseñanza de competencias genéricas. Recuperado de http://docencia.udec.cl/unidd/images/stories/ documentos/Modelo_de_Ensenianza.pdf

Vallejo, M., \& Molina, J. (2014). La evaluación auténtica de los procesos educativos. Revista Iberoamericana de Educación, (64), 11-25.

Velásquez, M., Arellanez, J., \& Martínez, A. (2012). Asertividad y consumo de drogas en estudiantes mexicanos. Acta Colombiana de Psicología, 15(1), 131-141.

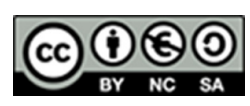

Esta obra está bajo una licencia de Creative Commons Reconocimiento

- NoComercial - CompartirIgual (by-nc-sa) 